\title{
Chiral symmetry breaking in $d=3$ NJL model in external gravitational and magnetic fields
}

\author{
D.M. Gitman \\ Instituto de Física, Universidade de São Paulo \\ Caixa Postal 66318, 05389-970-São Paulo, S.P., Brasil \\ S.D. Odintsov \\ Dept. of Math.Analys., Tomsk Pedagogical University \\ 634041 Tomsk, Russia, and Dept. ECM, Fac. de Fisica \\ Universidad de Barcelona, Diagonal 647, 08028 Barcelona, Spain \\ Yu.I. Shil'nov \\ Dept. Theor. Phys., Kharkov State University \\ Svobody Sq. 4, Kharkov 31007r, Ukraine
}

(October 5, 2018)

\begin{abstract}
The phase structure of $d=3$ Nambu-Jona-Lasinio model in curved spacetime with magnetic field is investigated in the leading order of the $1 / N$-expansion and in linear curvature approximation (an external magnetic field is treated exactly). The possibility of the chiral symmetry breaking under the combined action of the external gravitational and magnetic fields is shown explicitly. At some circumstances the chiral symmetry may be restored due to the compensation of the magnetic field by the gravitational field.
\end{abstract}

Typeset using REVTEX 
$d=3$ NJL model may be very useful laboratory for the study of dynamical symmetry breaking and composite bound states. This model is known to be renormalizable in $1 / N$ expansion and it has quite rich phase structure. In principle, such a model may be helpful as some high-temperature phase of the corresponding $d=4$ theory considered as kind of the effective theory for QCD.

Recently, phase structure of $d=3$ NJL model has been investigated in an external gravitational field [2,3] and in an external magnetic field [4 6, 12] (and references therein). It has been pointed out that magnetic field (for a general introduction to the theory in an external electromagnetic field see [7]) usually supports the chiral symmetry breaking. On the same time, for such a model on $S_{3}$ or $H_{3}$ spaces one can show [3] that chiral (flavor) symmetry is always broken in $H_{3}$ (hyperbolic space) while it can be restored for some values of the curvature on $S_{3}$ background.

Having in mind the possible cosmological applications of such considerations $[8]$ and the possibility of the existence of large primordial magnetic fields at the early Universe it would be of interest to discuss the combined effect of gravitational and magnetic fields to phase structure. That will be the purpose of the present letter.

We will start first from $d=3$ four-fermion model in curved spacetime:

$$
L=\bar{\Psi}^{i} \gamma^{\mu}(x) \nabla_{\mu} \Psi^{i}-\frac{N \sigma^{2}}{2 \lambda}-\sigma \bar{\Psi}^{i} \Psi^{i},
$$

where $i=1, \ldots, N, \quad N$ is number of fermion species, $\gamma^{\mu}(x)$ are curved $\gamma$-matrices and as usually the auxiliary scalar field $\sigma$ is introduced. We work in linear curvature approximation (keeping only linear curvature terms in the leading correction to effective potential) and also in $1 / N$-expansion. Then it is convenient to use the local momentum representation of the fermion propagator in curved spacetime in the form discussed in $[8]$.

Integrating in the functional integral over fermion fields in the standard way, one can get the effective potential as following $[8]$ :

$$
V(\sigma)=\frac{\sigma^{2}}{2 \lambda}+i \operatorname{Sp} \ln \left[i \gamma^{\mu}(x) \nabla_{\mu}-\sigma\right] .
$$


It is convenient to work in terms of the derivative from $V(\sigma)$. Then, using the local momentum representation of the propagator, we get

$$
\begin{aligned}
V^{\prime}(\sigma) & =\frac{\sigma}{\lambda}-i \operatorname{Sp} \int \frac{d^{3} k}{(2 \pi)^{3}}\left[\frac{\hat{k}+\sigma}{k^{2}-\sigma^{2}}-\frac{R}{12} \frac{\hat{k}+\sigma}{\left(k^{2}-\sigma^{2}\right)^{2}}\right. \\
& \left.+\frac{2}{3} R_{\mu \nu} k^{\mu} k^{\nu} \frac{(\hat{k}+\sigma)}{\left(k^{2}-\sigma^{2}\right)^{3}}-\frac{1}{2} \gamma^{a} \sigma^{c d} R_{c d a \mu} \frac{k^{\mu}}{\left(k^{2}-\sigma^{2}\right)^{2}}\right]
\end{aligned}
$$

where $\hat{k}=\gamma^{\mu} k_{\mu}$.

Note that in the leading order contribution to effective potential (2) $N$ has been factored out.

Making use of Wick rotation and calculating the trace in (3), we obtain

$$
\begin{aligned}
V^{\prime}(\sigma) & =\frac{\sigma}{\lambda}+\frac{2 \sigma}{\pi^{2}} \int_{0}^{\infty} k^{2} d k\left[-\frac{1}{k^{2}+\sigma^{2}}-\frac{R}{12} \frac{1}{\left(k^{2}+\sigma^{2}\right)^{2}}+\frac{2}{9} R \frac{k^{2}}{\left(k^{2}+\sigma^{2}\right)^{3}}\right] \\
& =\frac{\sigma}{\lambda}+\frac{\sigma}{2 \pi^{3 / 2}} \int_{1 / \Lambda^{2}}^{\infty} d s \exp \left(-s \sigma^{2}\right)\left(-s^{-3 / 2}+\frac{R}{12} s^{-1 / 2}\right) .
\end{aligned}
$$

In derivation of eq. (4) we have used the expression for proper-time representation

$$
A^{-\nu}=\frac{1}{\Gamma(\nu)} \int_{0}^{\infty} d s s^{\nu-1} e^{-s A}
$$

and after that, the ultraviolet proper-time cut-off $\Lambda^{2}$ has been introduced in the low limit of proper-time integral. Note that in [2] another cut-off (introduced as upper limit of momentum integrals) has been used.

Performing the integration over $s$ and over $\sigma$, we get:

$$
\begin{aligned}
V(\sigma) & =\frac{\sigma^{2}}{2 \lambda}+\frac{1}{6 \pi^{3 / 2}}\left[\Lambda^{3} \exp \left(-\frac{\sigma^{2}}{\Lambda^{2}}\right)-2 \sigma^{2} \Lambda \exp \left(-\frac{\sigma^{2}}{\Lambda^{2}}\right)+2 \sqrt{\pi} \sigma^{3} \operatorname{erfc}\left(\frac{\sigma}{\Lambda}\right)\right. \\
& \left.-\frac{R}{4}\left(\Lambda \exp \left(-\frac{\sigma^{2}}{\Lambda^{2}}\right)-\sqrt{\pi} \sigma \operatorname{erfc}\left(\frac{\sigma}{\Lambda}\right)\right)\right]
\end{aligned}
$$

where $\operatorname{erfc}(x)=\frac{2}{\sqrt{\pi}} \int_{x}^{\infty} e^{-t^{2}} d t$. Hence, we obtained the effective potential in $d=3 \mathrm{NJL}$ model in curved spacetime using proper-time cut-off.

The renormalized effective potential may be found in the limit $\lambda \rightarrow \infty$ as following:

$$
V(\sigma)=\frac{\sigma^{2}}{2 \lambda_{R}}+\frac{|\sigma|^{3}}{3 \pi}+\frac{R|\sigma|}{24 \pi},
$$


where

$$
\frac{1}{\lambda_{R}}=\frac{1}{\lambda}-\frac{\Lambda}{\pi^{3 / 2}}
$$

Let us analyse now the phase structure of the potential (7). In the absence of curvature, $R=0$, for $\lambda_{R}>0$ the minimum of the potential $(7)$ is given by $\sigma=0$. Hence, there is no chiral symmetry breaking. For $\lambda_{R}<0$ the chiral symmetry is broken, the dynamically generated fermion mass is given as

$$
m \equiv \sigma_{\min }=-\frac{\pi}{\lambda_{R}}
$$

When the curvature is not zero we find the following picture. Let first $\lambda_{R}>0$. Then the ground state (and dynamically generated mass) is defined by

$$
m \equiv \sigma_{\min }=-\frac{\pi}{2 \lambda_{R}}+\frac{1}{2} \sqrt{\frac{\pi^{2}}{\lambda_{R}^{2}}-\frac{R}{6}} .
$$

One can see that unlike the case of flat space for positive $\lambda_{R}$ we have the chiral symmetry breaking. Moreover, as it follows from [10] we see the possibility of curvature-induced phase transitions. The critical curvature is given by $R_{c}=0$ (flat space). For negative curvature, $R<R_{c}=0$, we observe the chiral symmetry breaking, while for positive curvatures symmetry is not broken.

For negative four-fermion coupling constant $\lambda<0$ we get the following ground state:

$$
\sigma_{\min }=-\frac{\pi}{2 \lambda_{R}}-\frac{1}{2} \sqrt{\frac{\pi^{2}}{\lambda_{R}^{2}}-\frac{R}{6}} .
$$

The critical curvature is defined by the condition: $R_{c}=\frac{6 \pi^{2}}{\lambda_{R}^{2}}$. Between $0<R \leq R_{c}$ the chiral symmetry is broken.

Now, let us discuss the situation when NJL model (11) is considered in curved spacetime with external magnetic field. That means that spinor covariant derivative also contains the electromagnetic piece. Treating the magnetic field exactly [9], one can easily find the effective potential for the model (1]). Considering mow NJL model in curved spacetime with magnetic field, we again work in linear curvature approximation as above (but making no 
approximations for an external magnetic field). Moreover, we take into account only leading contribution on curvature which doesn't depend on magnetic field, i.e. the contribution discussed above. (One can show that for not a very large magnetic field the curvature correction depending explicitly from magnetic field is not essential). Then, using the eq. (7) and the results of the calculation of $d=3$ NJL effective potential in an external magnetic field [曰, 6], one can get:

$$
\begin{aligned}
V & =\frac{\sigma^{2}}{2 \lambda_{R}}+\frac{|\sigma|^{3}}{3 \pi}+\frac{R|\sigma|}{24 \pi}+\frac{e H}{4 \pi^{3 / 2}} \int_{0}^{\infty} \frac{d s}{s^{3 / 2}} e^{-s \sigma^{2}} \\
& \times\left[\operatorname{coth}(e H s)-\frac{1}{e H s}\right]
\end{aligned}
$$

where $H$ is magnetic field and $e$ is electric charge.

The renormalized effective potential (12) may be represented as following

$$
V=\frac{\sigma^{2}}{2 \lambda_{R}}+\frac{R|\sigma|}{24 \pi}+\frac{e H|\sigma|}{2 \pi}-\frac{(2 e H)^{3 / 2}}{2 \pi} \zeta\left(-\frac{1}{2}, \frac{\sigma^{2}}{2 e H}\right),
$$

where properties of generalized zeta-function may be found in [10].

Working in frames of our approximation and considering also $e H \rightarrow 0$, we find that for positive $\lambda_{R}$

$$
\sigma_{\min } \simeq \frac{\lambda_{R}}{\pi}\left(\frac{e H}{2}-\frac{R}{24}\right)
$$

Hence, for $R=0$ chiral symmetry breaking due to magnetic field occurs. Negative curvature increases chiral symmetry breaking. However, positive curvature acts against CSB. On the critical line of phase diagram

$$
\frac{R}{12} \simeq e H
$$

the restoration of chiral symmetry breaking occurs. Hence, in this example magnetic field and gravitational field act in the opposite directions with respect to chiral symmetry breaking. Gravity tends to restore the chiral symmetry while magnetic field tends to break it. On the critical line both effects are compensated and there is no chiral symmetry breaking

For $\lambda_{R}<0, e H \rightarrow 0$ we get 


$$
\sigma_{\min }=\sigma_{\min }^{H=0}\left[1+\frac{(e H)^{2}}{12\left(\sigma_{\min }^{H=0}\right)^{4}}+\ldots\right] \text {, }
$$

where $\sigma_{\min }^{H=0}$ is given by the expression (11) (compare with [6]).

In summary, we studied $d=3$ NJL model in curved spacetime with magnetic field. The combined effect of gravitational and magnetic fields may lead to quite rich phase structure. In particular, magnetic field always breaks chiral symmetry. However, in curved spacetime with positive curvature the external gravitational field may lead to screening of magnetic field and restoration of chiral symmetry as it is shown above. We expect that this new effect should be relevant for cosmological applications, for example, in the consideration of GUTs and SM reformulated as NJL model [11] in the early Universe with primordial magnetic fields.

SDO would like to thank D. Amati for kind hospitality in SISSA, Trieste where this work has been completed. 


\section{REFERENCES}

[1] Y. Nambu and G.Jona-Lasinio, Phys. Rev. 122 (1961) 345

[2] E. Elizalde, S.D. Odintsov and Yu.I. Shil'nov, Mod. Phys. Lett. A9 (1994) 913

[3] E. Elizalde, S. Leseduarte, S.D. Odintsov and Yu.I. Shil'nov, Phys. Rev. D (1996), to appear; T. Inagaki, S. Mukaigawa and T. Muta, Phys. Rev. D52 (1995) R4267

[4] I.V. Krive and S.A. Naftulin, Phys. Rev. D46 (1992) 2737

[5] D. Cangemi, E.D'Hoker and G.V. Dunne, Phys. Rev. D51 (1995) 2513; A. Das and M. Hott, hep-th/9504086

[6] A.S. Vshivtsev, B.V. Magnitski and K.G. Klimenko, JETP Lett. 62 (1995) 283; K.G. Klimenko, Z. Phys. C54 (1992) 323

[7] E.S. Fradkin, D.M. Gitman and Sh.M. Shvartsman, Quantum Electrodynamics with Unstable Vacuum, (Springer-Verlag, Berlin, 1991)

[8] T. Inagaki, T. Muta and S.D. Odintsov, Mod. Phys. Lett. A8 (1993) 2117

[9] V.G. Bagrov and D.M. Gitman, Exact Solutions of Relativistic Wave Equations, (Kluwer Ac. Publishers, Dordrecht, 1990)

[10] E. Elizalde et al, Zeta-regularization with Applications, (World-Sci., Singapore, 1994)

[11] W. Bardeen, C. Hill and M. Lindner, Phys. Rev. D41 (1990) 1647

[12] V. Gusynin, V. Miransky and I. Shovkovy, Phys. Rev. Lett. 73 (1994) 3499 